Profesor Titular del Departamento de Pintura. Facultad de Bellas Artes, VELOSO

\title{
Arte asociacionista cubano para un fin de siglo: artivismo, performance y revolución
}




\section{CUBAN ASSOCIATIVE ART FOR THE END OF THE CENTURY: ARTIVISM, PERFORMANCE AND REVOLUTION}

\section{ABSTRACT}

This article analyzes some of the most significant groups of artists of the exuberant eighties in Cuba. Among the great variety of groups that appeared in this period, we are going to focus on those that, with greater clarity, use performance as a fundamental medium. This use of an ephemeral and unconventional discipline will reinforce the ties between the community of artists and the Cuban people at large, at a time when many still considered it possible to solve the many failures of the system through spontaneous and disinterested art. Pursuing this objective, the artists held firm to the hope that something could change and, sure of this conviction, they promoted collaborative work such as had not been seen since the historical avant-gardes in Cuba. After the devastating economic and social crisis of the 1990s, hope started to disappear. The arrival of the new century perpetuated hardship and managed to squander the former spirit of solidarity - not only among artists - but also among an important part of society. However, it seems correct to conclude that the current tense political context can function as a deterrent of disunity and individualism. The important popular protests against the government that took place on July 11, 2021, have caused a large number of creators, intellectuals and people outside the artistic field to decide to work again in community.

\section{Keywords}

Artivism; performance; Cuba; Twentieth century

\section{RESUMEN}

El presente artículo propone analizar algunos de los grupos de artistas más significativos de la explosiva década de los ochenta en Cuba. Dentro de la gran variedad de colectivos que aparecen en este periodo, vamos a centrarnos en aquellos que, con mayor claridad, utilizan la performance como medio fundamental. Este uso de una disciplina efímera y poco convencional reforzará los lazos de unión entre la comunidad de artistas y el pueblo en un momento en el que todavía se veía posible solventar los numerosos fallos del sistema a través de un arte espontáneo y desinteresado. Persiguiendo este objetivo, los artistas mantuvieron firme la esperanza de que algo podía cambiar y, seguros de esta convicción, potenciaron el trabajo colaborativo como no se había visto desde las vanguardias históricas en Cuba. Tras la devastadora crisis económica y de valores de los noventa, la esperanza desaparece. La llegada del nuevo siglo perpetuó la penuria y consiguió dilapidar el otrora espíritu grupal y solidario -no sólo de los artistas- sino de una parte importante de la sociedad. Sin embargo, parece acertado concluir que el tenso contexto político actual anima a sortear la desunión y el individualismo. Las importantes protestas populares contra el gobierno que tuvieron lugar el 11 de julio de 2021, han propiciado que una nutrida representación de creadores, intelectuales y personas ajenas al campo artístico hayan decidido trabajar nuevamente en comunidad.

Palabras clave

Artivismo; performance; Cuba; Siglo XX 


\section{INTRODUCCIÓN}

Todo análisis que pretenda investigar el intrincado contexto artístico de la Cuba posrevolucionaria debe partir de la experiencia in situ convirtiendo el trabajo de campo en una labor inexcusable. Teniendo esta norma como premisa, hemos querido incorporar a nuestra investigación la herencia multidisciplinar de los Cultural Studies, integrando áreas de conocimiento como las ciencias políticas y económicas, la sociología o la antropología. Esta metodología híbrida parece ser la más indicada para nuestro objeto de estudio que, como sabemos, está modelado por la influencia que ejerce su marco político, su pasado colonial o la compleja problemática socioeconómica de la periferia. Con frecuencia hemos encontrado el mismo abordaje metodológico entre los especialistas -tanto cubanos como extranjeros- que investigan este periodo del arte de la isla. Voces representativas como Magaly Espinosa, Ivan de la Nuez, Luis Camintzer, Gerardo Mosquera o Kevin Power serán referentes importantes al aportar un enfoque que se alimenta también del posestructuralismo o de un necesario debate teórico de corte poscolonial. Al lado de estas estrategias, nos gustaría subrayar que, un número considerable de los estudios sobre el arte cubano de la Revolución encuentran en la división por décadas una útil herramienta para intentar ordenar un complejo y heterogéneo capítulo de la historia reciente. Como es lógico, estas divisiones no se rigen por la precisión del calendario, sino que obedecen a un continuo fluir que nos permite relacionar hechos y generaciones. Sin embargo, el azar ha querido que esos ciclos vengan enmarcados por acontecimientos realmente trascendentales sucedidos en los años iniciales de cada decenio; acontecimientos que han tenido capacidad para transformar la política, la sociedad y, en consecuencia, el arte mismo. Por ello -siguiendo la práctica investigadora de conocidos y ya nombrados especialistas en la materia- será frecuente encontrar en nuestro análisis segmentos temporales que nos ayudarán a contextualizar las diferentes etapas de la temática central que nos ocupa.

El estudio que ahora comienza se organiza y estructura en relación a estos seis objetivos fundamentales: valorar si el contexto de los años ochenta ha propiciado la aparición de un asociacionismo artístico con capacidad para influir políticamente en entornos principalmente urbanos, confirmar si la irrupción de nuevas corrientes pedagógicas en la enseñanza del arte han sido importantes en la formación de estos colectivos, analizar por qué la performance se constituye como uno de los medios más utilizados entre estos grupos, sopesar la influencia de la censura oficial en el arte asociacionista de finales de siglo en Cuba, averiguar si la irrupción del mercado internacional y una voraz crisis económica fueron los factores responsables de la caída del arte grupal cubano y, por último, ratificar si los recientes acontecimientos de protesta popular del 11 de Julio de 2021 han podido funcionar como un poderoso detonante para resucitar la lucha colectiva de la clase artística e intelectual en Cuba. La consecución de estos objetivos aconseja detenernos en el intrincado marco político y sociocultural de la Cuba posrevolucionaria, en el contexto artístico cubano de los ochenta, en la incursión de la performance en los colectivos de arte cubano finisecular, en las pedagogías críticas del arte o en el análisis de la producción artística de algunas de las agrupaciones más representativas de ese momento. 
El 1 de enero de 1959 es una de esas fechas que merecen ser recordadas. En este significativo día, el dictador Fulgencio Batista abandona la Isla. Tan sólo una semana más tarde, Castro y sus tropas marchan por la ciudad de La Habana proclamando el triunfo de la Revolución. Cuba deja atrás una época marcada por una oscura y represiva dictadura donde un voraz capitalismo condenaba a una gran parte de la población a sufrir las consecuencias de una injusticia social que normativizaba la pobreza en amplios sectores de la población. Estos primeros años del triunfo revolucionario supusieron una puerta abierta a la esperanza para muchos. Sin embargo, también han significado una dolorosa experiencia para quienes no aceptaban las directrices de un férreo régimen que imponía los principios políticos de la Revolución por encima de cualquier cosa. Esta mirada unidireccional priorizaba también el carácter ideológico de la obra de arte como el único valor que justificaba su existencia. Como bien apunta Iván de la Nuez (1995), la política cultural de la Revolución Cubana se ha basado en una estructura fuertemente jerarquizada donde el intelectual actuaba dentro de unos márgenes dictados por el propio sistema. La conocida sentencia pronunciada por Castro en el año 1961 «dentro de la Revolución, todo; contra la Revolución, nada» (Castro, 1961/1987, p.28) sintetiza a la perfección la posición oficial del sistema político revolucionario en relación con el arte y la cultura durante estos primeros años. Según la percepción del joven gobierno de Castro, un arte desviado de los principios que marcaba la Revolución aumentaría la inestabilidad: una peligrosa inestabilidad alimentada por acontecimientos como Bahía de Cochinos, la conocida Crisis de los misiles o el asesinato de Ernesto Guevara El Che en Bolivia. Para combatir esta inseguridad, el Estado cubano blinda el sistema e intenta así hacer frente a las presiones de las potencias extranjeras que veían como su situación geopolítica perdía fuerza en la zona. En ese blindaje, el arte y la cultura comenzaron a perder autonomía presionados por un firme control gubernamental. El periodo inicial de la década siguiente, conocido como Quinquenio Gris (Fornet, 2007), dibuja una de las caras más opacas del régimen. La persecución de la cultura no oficial era constante. El arte continuaba considerándose como una práctica para construir una nueva Cuba socialista donde no se contemplaba ninguna traición a la norma. Tras esta dura represión institucional, llegaron los años ochenta marcados por una cierta estabilidad económica y una menor intensidad en la censura contra el arte. La comunidad de artistas cubanos desarrolla su trabajo fuera de la presión del mercado amparándose en las numerosas becas institucionales que les permitían salir de la Isla y establecer contactos en los grandes centros de arte internacionales. En esa época, nadie vaticinaba la caída de los países de la órbita socialista en Europa y las drásticas consecuencias que la Perestroika tendría en Cuba.

Los noventa se iniciaron con una brutal crisis económica. La Isla está más aislada que nunca. Cuba observa como su economía cae en picado sin los beneficios que le aportaba el comercio con países ideológicamente afines. Además, el persistente bloqueo estadounidense no facilita la recuperación económica del país (Alzugaray, 2012). El politólogo chileno Andrés Benavente Urbina confirma estas afirmaciones que explican el inicio de una de las crisis más importantes que azotó al país en la segunda mitad del siglo XX: "Los mercados de los países de Europa del Este se le han cerrado para sus productos y, a su vez, dichos países ya no prestan a Cuba la asistencia financiera, tecnológica y logística-militar» (1990, p.161). La situación se agrava porque, como apunta Mesa-Lago, el modelo cubano no es capaz de generar un crecimiento económico que perdure en el tiempo, sus exportaciones son escasas y unidireccionales y siguen siendo altamente dependientes del exterior, lo que provoca un elevado volumen de 
importaciones (2005). Dado este panorama, muchas eran las voces que vaticinaban la caída del régimen comunista en Cuba. Sin embargo, lejos de esta posibilidad, el Estado cubano decide priorizar el dogma revolucionario y correr el riesgo de sufrir una debacle general del sistema con dramáticas consecuencias sobre la población. Lamentablemente, este riesgo se acaba haciendo realidad y las carencias extremas se hicieron visibles durante lo que se ha conocido como Periodo Especial en Tiempos de Paz (Mesa-Lago, 2005). La crisis se dejó notar en todos los ámbitos de la vida en la isla y mantuvo una incidencia muy severa durante los años que van desde 1991 a 1994 (Power, 1999). Uno de los efectos más importantes para la cultura fue el goteo incesante de exilados que acabó desangrando a la clase artística e intelectual (De la Nuez, 2002). Muchos de los protagonistas del Renacimiento Cubano (Camnitzer, 1994) tuvieron que abandonar el país provocando un gran desánimo en la generación de artistas de finales del siglo XX que veían, impotentes, como sus referentes buscaban nuevos lugares donde poder vivir y desarrollar su carrera profesional. Esta huida en masa de creadores e intelectuales dejó también graves secuelas en el Instituto Superior de Arte $^{1}$ de La Habana, una institución que ha funcionado como motor fundamental para la formación de los colectivos artísticos a partir de los años ochenta. La emigración de una representativa parte de su profesorado afectó al cuadro docente provocando una improvisada entrada de profesores que eran recién egresados de la propia institución. A pesar de su entusiasmo, este nuevo cuadro de educadores no supo cubrir el hueco dejado por sus antecesores. Al lado de este demoledor paisaje, no debemos olvidar que los noventa trajeron, además, un repunte importante de la censura oficial contra el arte. No procede elaborar ahora un listado de exposiciones clausuradas, performances abortadas u obras de teatro que precipitaron el final de sus representaciones porque sería muy extenso. Valga recordar que la represión gubernamental contra el arte y la cultura en Cuba es cíclica y se aplica con una violencia que, aunque varía en intensidad, se mantiene constante (Suazo, 2018). Las consecuencias de la firma del polémico decreto 349/2018 (González, 2018) o las recientes detenciones provocadas tras las protestas del Movimiento San Isidro (De la Fuente, 2021) o del $27 \mathrm{~N}$, así lo certifican.

Los años que sucedieron al referenciado Periodo Especial en Tiempos de Paz se caracterizaron por una escasez continuada de productos básicos para la vida diaria. Esta escasez incluía la comida, ropa, medicamentos o materiales imprescindibles para mantener funcionando el sistema educativo, la red de transporte urbano e interurbano o el otrora pujante sistema de salud. El pueblo de Cuba experimenta lo que la socióloga Mayra Espina define como una crisis profunda de la vida cotidiana. Con muy buen criterio, Espina nos advierte que la acelerada insatisfacción de las necesidades básicas conlleva la desestructuración de la población y del sistema (2001, p.62). La crisis inundó también el campo artístico y cultural facilitando que el mercado del arte internacional se aprovechase de la compra a precios bajos. Consecuencia de la necesidad, el artista cubano del nuevo siglo primará el beneficio individual en aras de unos ingresos estables y de su proyección en la esfera internacional. Este giro desplazará el interés por lo colectivo hacia un mayor protagonismo de la carrera individual provocando una llamativa disminución de los numerosos grupos de artistas que poblaban la escena cultural cubana de la década de los ochenta y buena parte de los noventa (Weiss, 2011). La irrupción del mercado ha proporcionado un mejor nivel de vida a una minoría, pero ha terminado con el característico espíritu de colaboración de décadas pasadas. Esta situación ha propiciado que el arte contemporáneo cubano se encuentre, en estos momentos, en una fase sin grandes sobresaltos, participando activamente y sin complejos del sistema del mercado internacional. Gracias a ello, un número importante de artistas ha conseguido una mayor estabilidad económica, pero, como contrapartida sus obras adolecen de una falta de frescura y originalidad que, bajo nuestro punto 
de vista, no les beneficia. En ese sentido, la plástica cubana actual lleva algunos años mostrando una cara que Mosquera ya vaticinaba a finales de los noventa: «(...) el oportunismo de jugar con la crítica social a manera de un tema de moda, o aún como rasgo de un Cuban style, satisfaciendo expectativas cliché» (1999, p.20). Coincidiendo con Mosquera, Orlando Justo advierte que el artista cubano del siglo XXI está más preocupado por convertir su obra en un producto vendible, que por la propia obra en sí (2020). En paralelo a esta disfuncionalidad del arte, vemos surgir -ya entrado el nuevo siglo- un renovado compromiso político en asociaciones como el Instituto de Artivismo Hannah Arendt (INSTAR), o los ya referenciados $27 N^{2}$ y el Movimiento San Isidro (MSI). Sus reivindicaciones anticipan el espíritu de las masivas protestas populares contra el gobierno cubano del 11 de julio de 2021, llevadas a cabo en diferentes pueblos y ciudades de la Isla (De la Nuez, 2021). Estos colectivos, recuperarán el espíritu de grupo a través de la lucha política directa. Sus acciones que en ocasiones adoptan el formato de performance o videoclip, van a ejecutarse también fuera de las disciplinas del arte haciendo uso de la protesta, la concentración o, incluso, la huelga de hambre. La irrupción de internet -sobre todo desde la segunda década de este siglo- les permite llegar a un público mucho más numeroso que en tiempos pasados. Quizás por ello, el poder se muestra muy preocupado por las consecuencias y actúa con mayor inclemencia, poniendo nuevamente en grave peligro la libertad de expresión y reunión en Cuba (Marrero, 2021).

\section{EL CONTEXTO ARTIVISTA DE LA CUBA DE LOS OCHENTA}

Aunque encontramos precedentes claros en algunos movimientos de la vanguardia europea de entreguerras o en la implicación política de la performance de los setenta del siglo pasado, el artivismo, como categoría epistemológica, comienza a ser popular en la década final del siglo $X X$. La práctica artística de estos colectivos (en su mayoría urbanos) surge como respuesta a la creciente insatisfacción social provocada por repetidas crisis de índole económico o ideológico. En sintonía con lo expresado por Delgado (2013), entendemos el artivismo como un asociacionismo artístico que intenta, en esencia, resignificar políticamente el espacio urbano y despertar la conciencia de las personas que habitan en ese contexto. Para ello, se vale con frecuencia de la performance, del sentido del humor, de la ironía, de una consciente renuncia a la autoría artística o de un rechazo hacia la institución. Junto a estas estrategias, el artivismo encuentra pleno sentido cuando, tal y como apunta Parramón, existe una «vinculación con la especificidad del lugar y el compromiso con la realidad» (2003, p. 51). La definición de activismo artístico planteada por Expósito, Vindel y Vidal ratifica nuestras anteriores afirmaciones al entender el activismo como un arte comprometido con la acción social que reacciona contra un vacío producto artístico defendido por el dogma de la modernidad (2012). El abundante arte asociacionista cubano de los ochenta sigue la estela del artivismo activo al comprometerse firmemente con su realidad política, al utilizar la performance como medio esencial de expresión, al vincularse a lo local para lanzar una llamada de atención a las múltiples debilidades del sistema o al actuar principalmente en el espacio público para conectar sin intermediarios con una audiencia muy receptiva.

El paisaje de los años ochenta se ha caracterizado por una apertura ideológica del gobierno hacia el arte y la cultura. Tras la creación en 1976 del Ministerio de Cultura, la política represiva de años anteriores parece bajar poco a poco de intensidad (Mosquera, 1991). Este cambio de rumbo se consolidará con la puesta en marcha del conocido Proceso de rectificación de errores abordado en el Tercer Congreso del Partido Comunista de Cuba celebrado en 1986 (Mesa-Lago, 
1991). En este momento, el poder daba la bienvenida a aquellos artistas cuya obra estuviese centrada en una revisión crítica del sistema. Así, aun considerando la censura un mal endémico en Cuba, la situación durante los ochenta experimenta un cambio de rumbo y permite un ambiente creativo liberado de muchos de los condicionantes que solo unos pocos años antes hubiesen puesto un límite claro a la labor de la comunidad artística. Nace un arte nuevo que apuesta por la comunicación mostrando con desparpajo estrategias que, en palabras de Kevin Power, ponen en primer plano herramientas como la «ironía, parodia, sarcasmo enojado, arte de la calle, performance, y la energía abrumadora de su juventud» (2006, p.1). Será precisamente esta energía desbordante otro de los factores que también ha permitido la explosión de manifestaciones culturales en otras disciplinas como el teatro, la danza, el cine o la literatura.

El ISA ha sido otro de los pilares fundamentales a la hora de construir lo que se ha conocido como el Arte de los ochenta en Cuba. La llegada al claustro docente de nuevos profesores como Flavio Garciandia o Consuelo Castañeda impulsará la crítica y el diálogo bajo una visión sociológica del arte dejando definitivamente atrás concepciones más arcaizantes ligadas a una enseñanza más tradicional (Mora, 2007). Esta institución se ha caracterizado por instaurar novedosas metodologías docentes donde se repasaban referentes teóricos de orden internacional al tiempo que se analizaba la influencia con lo vernáculo, el pasado colonial y la obvia relación con el contexto artístico caribeño y latinoamericano. Las directrices pedagógicas que, hacia la segunda mitad de los ochenta, marcaron una etapa más abierta en el ISA se basaban en comprender los diferentes procesos de la cultura contemporánea integrando todas las opciones expresivas, entender la práctica pedagógica del arte como un instrumento de diálogo con el estudiantado, integrar los diferentes discursos artísticos surgidos en el contexto social y crear un artista que no reduzca su propuesta a una obra sino que piense su trabajo como una experiencia cultural que no persigue lo puramente objetual (Álvarez, 2007). Junto a estas líneas de actuación, el Instituto se caracterizaba por aplicar una enseñanza horizontal que desbancaba las jerarquías académicas para priorizar una pedagogía personalizada que se adaptaba y acompañaba el proceso creativo de los estudiantes (Salort, 2010). Dentro de este proceso, la experimentación, tanto en el abordaje técnico como en la base conceptual, era considerada el motor esencial para llegar a conseguir una línea de trabajo autónoma, singular y coherente (Salort, 2018). Los docentes eran, además, artistas en activo que promovieron muchas de las experiencias grupales más interesantes del arte finisecular cubano, baste recordar la trayectoria de importantes grupos surgidos en el ISA como Galería DUPP (Desde una Pragmática Pedagógica) o Enema.

\section{NUEVOS MODELOS DE PRODUCCIÓN ARTíSTICA EN LA CUBA DE LA REVOLUCIÓN: COLECTIVOS, ASOCIACIONES Y OTRAS REUNIONES}

La antesala de la gran proliferación de grupos que tuvo lugar a partir de la mitad de la década de los ochenta vino de la mano de conocidas experiencias como Pintura Fresca o la tan nombrada exposición Volumen I. Esta significativa muestra, inaugurada en 1981, abre una nueva época al reunir a un conjunto de artistas que investigan otros planteamientos plásticos utilizando tanto una visión renovada de lenguajes ya aceptados, como apostando por la instalación o la performance (Mosquera, 1981). Sin embargo, lo que más nos llama la atención de la experiencia de Volumen I es su trabajo colaborativo. Tal y como apunta Camnitzer, la importancia verdadera de la exposición radica en el despertar de la conciencia de grupo que funcionará como ejemplo para otros colectivos que surgirán con posterioridad a esta famosa muestra (1994). Tras Volumen I el arte cubano abraza la creación en grupo recordando el espíritu de anteriores experiencias 
colectivas como la muestra Exposición de Arte Nuevo, inaugurada en 1927 o el conocido grupo Los Once, que han sido responsables de la llegada del expresionismo abstracto en la Cuba de la prolífica década de los cincuenta (Camnitzer, 1994). Volumen I, a pesar de articularse con piezas individuales, fue concebida como un todo destacando el valor de la obra no solamente como expresión individual, sino como parte de un conjunto que hablaba el mismo lenguaje. Entre las piezas de la muestra destacan abundantes registros y restos físicos de performances, algo que no era frecuente encontrar en las galerías de arte cubanas y que, sin duda, ha contribuido al conocimiento de las prácticas efímeras.

La gran cantidad de grupos que ven la luz en Cuba a partir de los primeros años de los ochenta resulta llamativa ${ }^{3}$ (Kocur, 2011). Dentro de esta diversidad, existen asociaciones como Eighteens, $4 \times 4$ o Punto que centraron su actividad en la puesta en marcha de una serie de exposiciones que no trascendían de la clásica exhibición de piezas individuales en una sala institucional. En realidad, muchas de estas experiencias se limitaban a comisariar colectivamente una muestra puntual sin desarrollar un trabajo comunitario sostenido en el tiempo. Es el caso de convocatorias como Arte en la Fábrica o el Proyecto Castillo de la Fuerza. Sin embargo, la irrupción de otras experiencias como Hexágono comenzaron a situar el arte cubano finisecular dentro de una dinámica grupal que tendrá una clara influencia en colectivos posteriores. Hexágono fue uno de los primeros colectivos importantes surgidos a inicios de los ochenta. A pesar de su corta actividad (desde 1982 a 1984) esta agrupación -formada por los artistas Humberto Castro, Sebastian Elizondo, Antonio Eligio "Tonel”, Consuelo Castañeda, Abigail García y María Elena Moreraconformó una de las primeras experiencias de creación colectiva tras la irrupción de Volumen I. Tal y como apunta Elvia Rosa Castro (2017), las obras de Hexágono mantienen la autoría de grupo sin establecer conexiones de estilo con el trabajo desarrollado por los integrantes a nivel individual. La actividad del grupo se asocia, fundamentalmente, con intervenciones artísticas en el entorno natural lo que les otorgará la etiqueta de ser el primer colectivo de land art cubano. Complementando la labor de Hexágono, la actividad del Grupo Puré ha tenido una importancia capital. Su trabajo, con una mirada comprometida hacia la realidad sociopolítica de Cuba, apostó -en palabras de Jana Cazalla- por enfatizar «lo escatológico, lo contradictorio y lo depauperado de la cultura popular como tratamiento de choque» (1995, p.214). Puré estaba integrado por Adriano Buergo, Lázaro Saavedra, Ciro Quintana, Ermy Tamaño y Ana Albertina Delgado. Entre todos ellos, destacamos la figura de Lázaro Saavedra que acabará siendo un docente destacado del ISA y alma mater del colectivo Enema.

Con posterioridad a estos intensos años la performance, el arte en colectividad y la autogestión no cesan. Sustentando esta idea, podemos afirmar que la profunda crisis de los noventa acabará potenciando la presencia de la performance grupal autogestionada debido, en parte, a su fácil abordaje, libre de los costosos materiales necesarios para el desarrollo de la obra objetual (Saavedra, 2014). Dentro de esta política de trabajo que apuesta por sacar partido de los mínimos recursos debemos recordar el Espacio Aglutinador, un organismo independiente que nace en un domicilio particular y que, desde 1994, se constituye como un importante punto de encuentro para la dinámica comunidad de artistas habanera. Allí se presentaron trabajos, con frecuencia centrados en la performance, que se desarrollaban fuera de la protección y de la presión institucional (Wilkinson, 2010). Aglutinador, creado por los artistas cubanos Sandra Ceballos y Ezequiel Suárez, continúa hoy en día su actividad quizás algo alejado del espíritu altruista y desinteresado que lo vio nacer; más interesado en ser una legítima fuente de ingresos para sus colaboradores y gestora (Fusco, 2017b). Al lado de este proyecto, conviene no olvidar otros como La Cátedra de Conducta iniciado en el año 2002. Esta experiencia, llevada a cabo 
por la artista Tania Bruguera en el Instituto Superior de Arte de la Habana, fue una aventura pedagógica con fértiles derivaciones hacia el arte no objetual (Espinosa, 2009). Los resultados de La Cátedra de Conducta nos confirman que fue algo más que un seminario académico al permitir la aparición de importantes proyectos de grupo generados a través del diálogo y la colaboración. Sin duda, el trabajo de la Cátedra ha sabido sintetizar la política pedagógica del propio ISA que, como ya hemos apuntado, ha priorizado la crítica colectiva, el diálogo y la colaboración entre estudiantes y profesores. Ya fuera de la capital, merece mención especial $L a$ Peña del Júcaro creada en Camagüey en el año 1995 (Ramírez, 2019). Esta asociación promueve la creación cultural tomando la figura de Martí como punto temático de partida. A lo largo de sus 25 años de trayectoria, ha albergado multitud de recitales poéticos, exposiciones y performances consiguiendo crear un fuerte sentimiento de comunidad entre vecinos y visitantes.

Además de este asociacionismo de raíz filantrópica, nos encontramos con otros tipos de corporaciones que, aunque apuestan por la gestión curatorial con fines abiertamente comerciales (González, 2017), acaban convirtiéndose en lugares de reunión y colaboración. Valga recordar los casos de espacios puestos en marcha por los propios artistas o por comisarios independientes. Estos son los lugares que han destacado desde el segundo decenio del nuevo siglo: Artista x Artista, coordinado por Carlos Garaicoa como espacio de exhibición y residencia para artistas extranjeros, también dedicado a la gestión de residencias internacionales para artistas cubanos; El Apartamento, lugar fundamentalmente para exhibición, dirigido por Cristian Gunding o la galería Taller Gorria, llevada por el gestor Adám Perugorria. Fuera de estas experiencias curatoriales, merece la pena destacar aquí la existencia de otras asociaciones en otros campos muy influyentes en lo social como, por ejemplo, la música. Al igual que Carlos Varela, Gente de Zona o Yotuel Romero, el grupo Porno para Ricardo (Figura 1) llegó a ser verdaderamente popular y realmente molesto para el gobierno. Sus letras y sus puestas en escenas -cercanas, en ocasiones, a la performance- destilaban una profunda crítica al sistema, lo que provocó una dura represión institucional que acabaría con una condena de cuatro años de prisión para Gorki Águila, líder del grupo y egresado del ISA (Fusco, 2017a). Considerando el trabajo de estos significativos ejemplos, nuestro estudio se dirige ahora hacia aquellos colectivos artísticos que desarrollaron procesos políticamente comprometidos en los que la performance en espacios públicos mantenía un protagonismo destacado. Esta orientación nos lleva a concentrar nuestro interés en el trabajo desarrollado por: Grupo Provisional, Arte Calle, Galería DUPP (Desde una Pragmática Pedagógica) y Enema. 


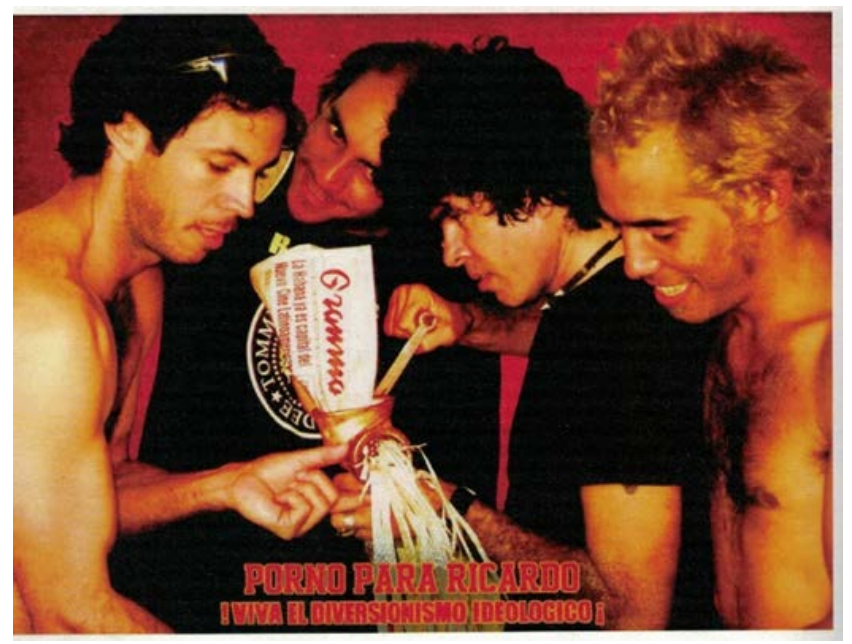

Figura 1. Folleto promocional del grupo Porno para Ricardo.

\subsection{Estudios de caso representativos}

El núcleo principal del Grupo Provisional lo formaron los artistas Carlos Rodríguez Cárdenas y Glexis Novoa desde 1986 a 1989. Segundo Planes fue otro de los colaboradores del colectivo en sus etapas iniciales. Con el paso del tiempo, Planes adopta una posición secundaria, aunque sigue participando de las discusiones del grupo y colaborando de forma puntual. El grupo surge y muere con cada proyecto, de ahí la elección de su nombre que podría hacer alusión a las vicisitudes que atravesaban en ese momento ciertos sectores de la plástica cubana. Para el Grupo Provisional las aportaciones de Volumen I fueron meramente una renovación estética. Críticos con el pasado y escépticos con el legado de sus predecesores, Cárdenas, Novoa y Planes perseguían algo más radical que dinamitase cierto inmovilismo político provocado por una Revolución que necesitaba una revisión urgente (Camnitzer, 1994). Las performances de este grupo -a menudo colaboraciones con Arte Calle- demostraban un gran sentido del humor apostando por una ácida sátira hacia lo institucional. En ocasiones muestran interés en la apropiación crítica del acervo cultural cubano dándole un giro a esa visión folclórica y superficial que utiliza la cultura como moneda de cambio frente a un poderoso extranjero que, con actitud prepotente, recoloniza la Isla de nuevo. Dentro de esta revisión crítica al pasado colonial, tomemos como ejemplo una significativa performance que Provisional llevó a cabo en el Museo Nacional de Cuba en 1988 durante una conferencia de Rauschenberg. Con un auditorio lleno, el grupo irrumpió con un cartel en el que se podía leer: "Very Good, Rauschenberg". Con este irónico asentimiento, el colectivo cuestionaba la actitud acrítica hacia discursos que, por el mero hecho de representar el centro hegemónico, se asimilan como un dogma inamovible que no puede ser cuestionado desde las voces de la periferia (Weiss, 2011).

Arte Calle desarrolla su actividad durante los años 1986 y 1990. Dentro del contexto que estamos analizando, quizás sus componentes han sido los que han demostrado un mayor contacto con las clases populares, sobre todo en núcleos urbanos. Una de sus premisas se basaba en el rechazo al objeto artístico favoreciendo un tipo de intervención más cercana a la performance y a lo procesual que evitaba los espacios sacralizados del arte como la galería o el museo 
(Cazalla, 1995). Siguiendo a Weiss (2011), podemos afirmar que estos jóvenes estudiantes se consideraban a sí mismos como terroristas del arte y priorizaban el impacto político de sus acciones por encima de cualquier regla formal. Entre sus muchas y polémicas intervenciones, Arte Calle ha irrumpido con máscaras antigás en una reunión de la Unión Nacional de Escritores y Artistas de Cuba (UNEAC) (Figura 2); ha convocado a diferentes personalidades de la cultura a una reunión que acabo siendo un encierro contra la voluntad de los presentes; o ha pintado murales con altas dosis de crítica política en las calles más céntricas de La Habana. Especialmente populares fueron estos murales (realizados en 1986) cuya firma AC (Arte Calle), fue rápidamente interpretada como un acrónimo que la población leía como "Abajo Castro". Al igual que en otras ocasiones, el propio contexto sociopolítico de Cuba hacía que estos murales fuesen mucho más que una simple pintura urbana. Además, las actividades de Arte Calle buscaban la colaboración lúdica del público. Un ejemplo significativo lo tenemos cuando, en el año 1988, Aldito Menéndez, polémico miembro del grupo, escribió la frase "Reviva la Revolu" y solicitó a los transeúntes una contribución económica para poder terminar la obra. Por una parte, esta performance de Menéndez ironizaba con el verdadero valor político de las consignas oficiales en Cuba y, por otra, advertía del escaso apoyo oficial al arte. El grupo no tuvo una plantilla estable porque con él colaboraban amigos del mundo de la música, el teatro o incluso vecinos de los barrios donde actuaban. A pesar de esta diversidad, podemos detectar un núcleo permanente en las figuras de Aldito Menéndez, Ofill Hechevarría, Ariel Serrano, Ernesto Leal, Pedro Vizcaíno y Eric Gómez Galán. Influidos por un cierto espíritu punk, reivindican el sustrato underground de la cultura periférica habanera (Fernández, 2003).

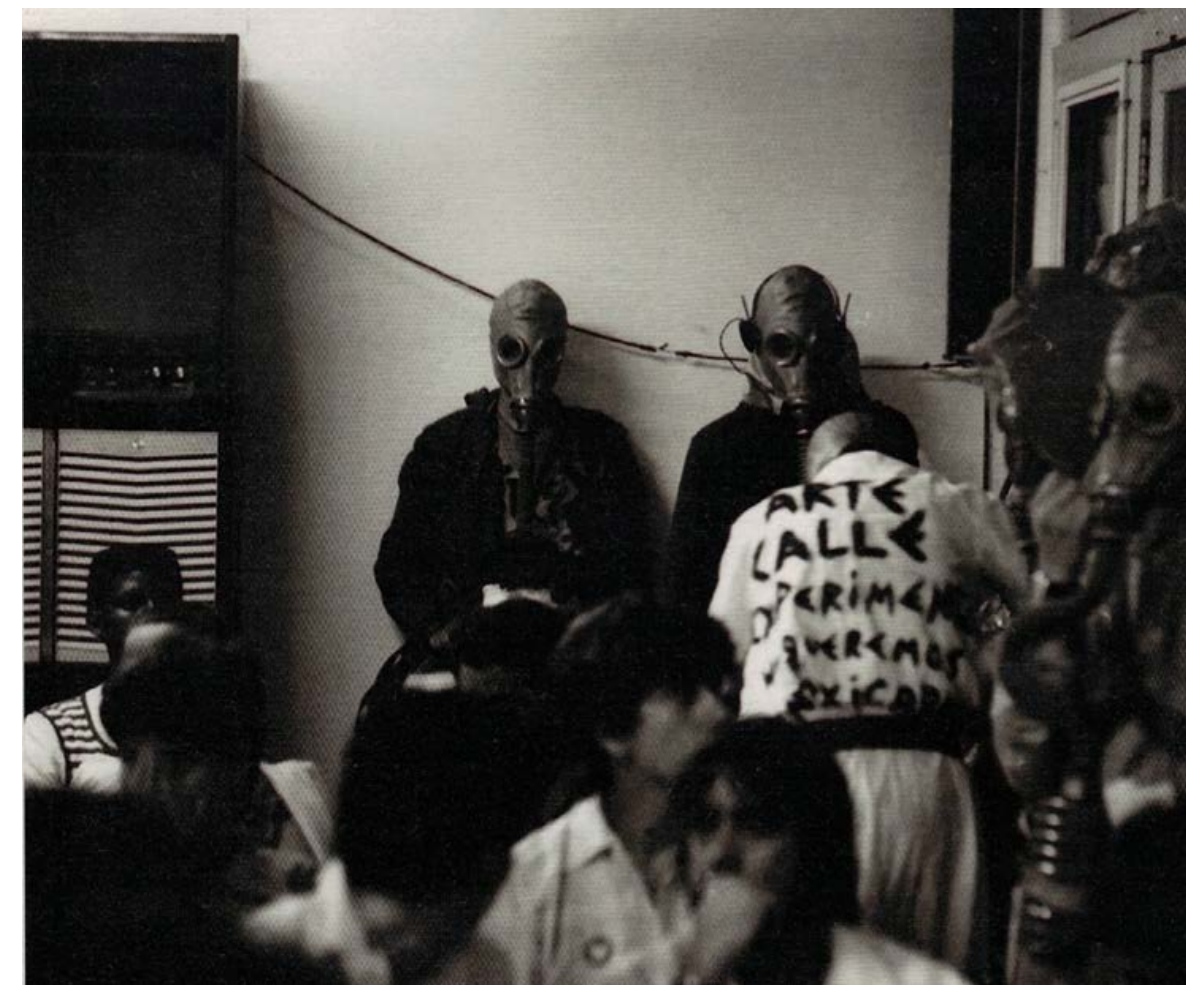

Figura 2. Arte calle. No queremos intoxicarnos, performance durante una conferencia en la UNEAC. 1987. Fotografía anónima. Archivo Arte Calle. 
Galería DUPP (Desde una Pragmática Pedagógica), se origina en el Instituto Superior de Arte de La Habana bajo el empuje del profesor Rene Francisco. El primer proyecto del grupo llevó por título La Casa Nacional y constituye uno de los mejores ejemplos de arte social de final de siglo pasado en Cuba. La performance, realizada de 1989 a 1990, se desarrolló en un edificio situado en el número 455 de la conocida calle Obispo en la ciudad de La Habana. En esta ocasión los miembros del grupo se ofrecieron a los vecinos para reparar, reformar o adaptar sus casas según sus deseos; incluso tenían cabida reformas de una naturaleza menos práctica, más inclinadas hacia lo emocional. "Las peticiones iban desde una mesa, un lavadero, hasta que les pintasen el techo; también un rincón poético. Hubo un hombre que solicitó un cuadro sobre un acontecimiento familiar" (Ojeda, 2001/2006, p.283). A través de estas peticiones, la performance demostraba como la tan manida conjunción entre arte y vida podía ser posible, adquiriendo un compromiso real con un barrio pobre que presentaba -y todavía presenta- muchas carencias. El trabajo de Galería DUPP mantuvo un cuestionamiento crítico hacia la institución arte y desmanteló la tradición clásica representada por un arte elitista ligado a la galería o al museo. Su trayectoria hizo de la performance su estrategia fundamental, apostando por una hacer de grupo que nuca abandonarán. Superando cualquier tipo de dinámica jerárquica, DUPP, optó por un planteamiento horizontal, dialogante y consensuado. Sus proyectos se derivaban de una puesta en común que evitaba la autoría individual. Así, el colectivo modificó el transcurso habitual de un conocido espacio comercial ( La Época) o revisitó las grutas de Jaruco donde Mendieta hizo una de sus series más celebradas. Precisamente, será tras este viaje cuando DUPP participa en el Primer Festival de Performance Ana Mendieta llevado a cabo en la Unión de Escritores y Artistas de Cuba (UNEAC) en 1998 y en el Segundo Festival de Performance, Pabellón del Vacío que tuvo lugar en el habanero Pabellón Cuba en 1999. La actividad de Galería DUPP atraviesa varias fases y se extiende hasta el año 2002. A pesar de que DUPP nos ha dejado una plantilla flexible de participantes, podemos advertir una serie de nombres que constituyen un centro más estable: Alexander Guerra, Beverly Mojena, David Sardiñas, Glenda León, Inti Hernández, Iván Capote, James Bonachea, JEFF, Juan Rivero, Yunior Mariño, Mayimbe, Michel Rives, Omar \& Duvier, René Francisco, Ruslán Torres, Wifredo Prieto y Yoán Capote.

Enema surge en los albores del nuevo siglo. Concretamente, su actividad comienza en el año 2000 y llegará -de forma continuada- hasta el año 2003. Al igual que el Grupo Puré, Galería DUPP o el Departamento de Intervenciones Públicas (DIP), su trayectoria se vincula al Instituto Superior de Arte de La Habana que funcionó como punto de encuentro y como motor del trabajo desarrollado por el colectivo. Como también ha sucedido en el caso de Galería DUPP, Enema toma impulso gracias al empuje de una comprometida actividad docente. La figura del artista y profesor Lázaro Saavedra ha sido clave en la formación del colectivo al estimular a un nutrido grupo de estudiantes que encontraron en el dialogo y la colaboración las herramientas esenciales para ponerse en marcha. Enema es uno de los grupos de arte cubano del nuevo siglo que con más consistencia y continuidad utiliza la performance en su trabajo (Vazquéz, 2003). La apropiación de performances históricas de la historia del arte se convertirá en una metodología frecuente. Así construyen versiones a partir de piezas como Two Stage Transfer Drawing de Oppenheim, Rope Piece de Teching Hsieh y Linda Montano o Breaking the ice de Marina Abramovic. Esta práctica apropiacionista -muy presente desde los ochenta en el arte cubano (Mosquera, 1998/2006a)- no constituye un mero ejercicio mimético, sino que, como apunta Saavedra (2014), responde a un lúcido ejercicio interpretativo que busca contextualizar el trabajo de los clásicos creando una nueva y compleja semántica para las performances que se desarrollan, en su mayor parte, en contextos urbanos. Ustedes sienten lo que ven, nosotros vemos (Figura 3 ) es, a nuestro juicio, una de las performances mejor resueltas del grupo. Este 
trabajo parte de una performance de Marina Abramovic en coautoría con Ulay titulada You see what you feel, I see de 1984. Para esta adaptación, Enema consultó solamente una fotografía. En Ustedes sienten lo que ven, nosotros vemos, observamos un andamio que los integrantes de Enema utilizarán para colgarse boca abajo formando una línea. Sus pies están atados al andamio con un trozo de sábana blanca. Permanecen en esa posición hasta que físicamente les resulta difícil seguir manteniendo la postura. Como en otras de sus performances, descubrimos un sutil uso de la metáfora que funcionará como detonante de un mensaje que, dentro del contexto cubano, se interpreta como una revisión crítica del sistema que podría darle la vuelta a lo más inamovible. Los integrantes de Enema fueron: Pavel Acosta, Daniel Beltrán, James Bonachea, Alejandro Cordobés, Zheina Couso, Nadieshzda Inda, Edgar Echevarría, Lino Fernández, Janler Méndez, Fabián Peña, Hanoi Pérez, Rubert Quintana, Lázaro Saavedra y Adrián Soca. Los rasgos más característicos del colectivo apuestan por el uso de la apropiación, la sencillez técnica, un acusado sentido del humor, la revisión crítica de los problemas que atañen a lo social, la preferencia por el espacio público y una fuerte conciencia de grupo que rechaza abiertamente la autoría individual.

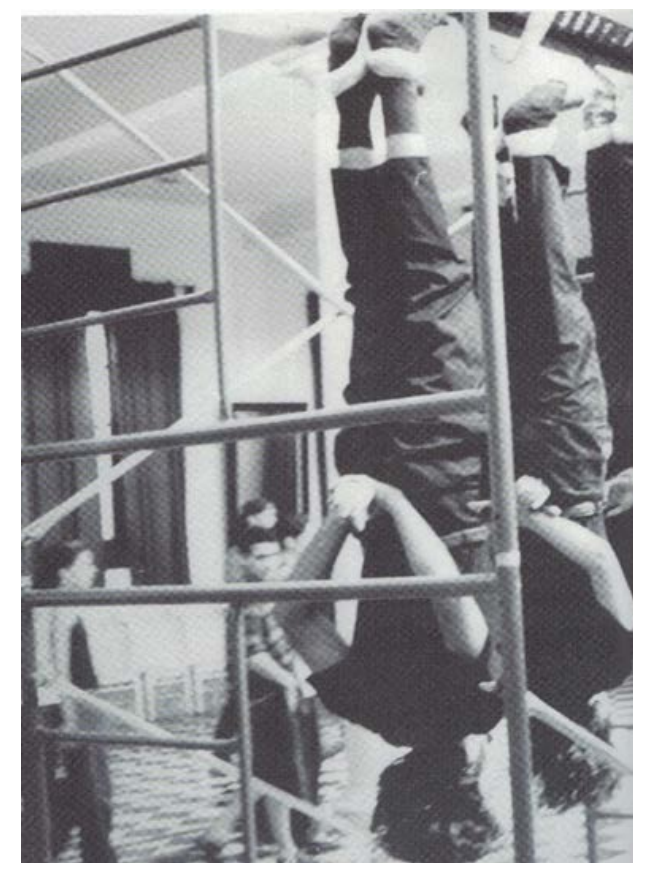

Figura 3. Enema, Ustedes sienten lo que ven, nosotros vemos. 2001, La Habana, Cuba. Fotografía anónima. Archivo fotográfico Enema. 
Los años ochenta del siglo pasado nos han dejado una gran proliferación de colectivos artísticos en Cuba. Alimentados por una frágil estabilidad, estos grupos surgen en un momento de tregua donde las tensiones políticas y económicas bajan su acostumbrada intensidad. Los artistas de este periodo gozan, sin saberlo, de un paréntesis de tranquilidad y del apoyo de organismos como el propio Ministerio de Cultura o el Instituto Superior de Arte de La Habana. Concretamente éste último ha funcionado como un punto de encuentro vital para la formación de muchas de estas agrupaciones que, conocedoras del temido Quinquenio Gris de los setenta, saben cómo aprovechar los nuevos aires pedagógicos que inundaban esta institución académica. Un optimista sentimiento de colectividad nace tras los muros del Instituto donde el diálogo, la colaboración y el entendimiento son las características vertebrales de estos años de aprendizaje. La disminución de la censura oficial, que no volverá a aumentar hasta finales de la década, fue otro de los factores que han favorecido la creación artística en este momento. Se produjo entonces un contexto muy favorable que solo se vio interrumpido cuando, la crisis de los noventa desestructura -de forma inesperada- la política, el arte y la sociedad de la época. A pesar de lo difícil de la situación, una compacta colectividad artística mantiene el trabajo colectivo y una clara función social del arte. En esta época de escasez profunda, la performance se convierte en el arma preferida por la comunidad artística. Su ductilidad y espontaneidad le permite adaptarse como ninguna otra técnica a la urgencia del momento. Brota un tipo de performance que decide no esclavizarse a sofisticados recursos técnicos y que prioriza el contacto directo con el pueblo para poner en marcha una oportuna revisión de las carencias del régimen. Esta irrupción de una performance combativa acabará consolidándose y, por consiguiente, molestando al poder, que aumentará significativamente la represión oficial. El dialogo entre artistas y autoridades se paraliza y la Revolución parece recuperar posiciones dogmáticas que nos recuerdan las peores épocas de censura de los primeros años del triunfo revolucionario. Simultáneamente, la interminable y demoledora crisis económica, la irrupción del mercado del arte internacional y una drástica (y comprensible) disminución de la camaradería entre los artistas, acabarán por apagar, poco a poco, el otrora potente asociacionismo cubano. La esperanza de que algo pueda cambiar se derrumba de manera definitiva.

Tras la desaparición de Enema, todavía podemos encontrar colectivos como, por ejemplo, El Grupo Trance, fundado en 2008. Pese a ello, el panorama asociacionista no volverá a ser el mismo ni tendrá el vigor de la práctica que arrasó durante las décadas de los ochenta y noventa. Aun así, no podemos negar que siguen existiendo agrupaciones muy activas en la Isla. Sin embargo, al analizar de cerca estos colectivos, percibimos que están integrados por personas de procedencias muy diversas y que incorporan a sociedad civil que ya no pertenece en exclusiva al campo artístico. Además, hemos observado que priorizan una acción política directa y que la represión del Estado contra su labor es, si cabe, más intensa. Estos movimientos, amplificados por la popularidad de las redes sociales a partir del segundo decenio de este siglo, exigen -entre otras reivindicaciones- plenas libertades y la instauración de un renovado y plural parlamento en Cuba. Las masivas movilizaciones populares contra el gobierno del pasado 11 de julio de 2021 sintetizan sus objetivos y, al mismo tiempo, evidencian que la práctica artística como herramienta crítica per se ya no es suficiente. 


\section{REFERENCIAS}

Álvarez, L. (2007). Memoria de nubes. En, A. Santana (Ed.). Nosotros, los más infieles: narraciones críticas sobre el arte cubano (1993-2005) (pp. 271-280). Murcia, España: Cendeac.

Alzugaray, C. (2012). Las (inexistentes) relaciones Cuba-Estados Unidos en tiempos de cambio. Nueva sociedad, (242), 139-148.

Benavente, A. (1990). Cuba y la reformulación del comunismo. Política. Revista de Ciencia Política, (24-25), 159-170.

Camnitzer, L. (1994). New art from Cuba. Austin: University of Texas Press.

Castro Ruz, F. (1987). Palabras a los intelectuales. En N. Sánchez y G. Fernández. (Eds.), Antología. Pensamiento y política cultural cubanos. (Vol. II, pp. 23-42). La Habana, Cuba: Pueblo y Educación. (Obra original publicada en 1961).

Castro, R.E. (2017). El objeto, la imagen y Equipo Hexágono: Redescubriendo un colectivo importante de los años 80. The archive. Cuban Art News. Cuban art and culture worldwide. Recuperado de https://cubanartnewsarchive.org/es/2017/05/30/ el-objeto-la-imagen-y-equipo-hexagono-redescubriendo-uncolectivo-importante-de-los-anos-80/

Cazalla Piñero, J.M. (1995). Arte Cubano de fin de siglo (Tesis doctoral no publicada). Universitat Politècnica de València (UPV), Valencia.

De la Fuente, A. (2021). Cuba: racismo y represión. El País (Opinión). Recuperado de https://elpais.com/ opinion/2021-06-03/cuba-racismo-y-represion.html?event_ log=oklogin?event_log=oklogin

De la Nuez, I. (1995). Al encuentro de los pasos perdidos. Los principios del arte y el arte de los principios en la Revolución Cubana (1959-1980). En, M. L. Borrás y A. Zaya. (comisarios), Cuba siglo XX. Modernidad y Sincretismo. (pp. 59-79). Cabildo de Gran Canaria, España: Ediciones Tabapress.

De la Nuez, I. (2002). El destierro de Calibán. Diáspora de la cultura cubana de los noventa en Europa. En, M. Gras. (comisaria), Atravesados. Deslizamientos de identidad y género. (pp. 63-67). Madrid, España: Decisión Gráfica. 
De la Nuez, I. (2021). Cuba, de la guerra fría a la guerra cultural. El País (Babelia). Recuperado de https://elpais.com/ babelia/2021-07-31/cuba-de-la-guerra-fria-a-la-guerracultural

Delgado, M. (2013). Artivismo y pospolítica. Sobre la estetización de las luchas sociales en contextos urbanos. Quaderns-e de I'Institut Català d'Antropologia, 2013, vol. 18, num. 2, p. 68-80.

Espina, M., Rodríguez, J. L., Triana, J., \& Hernández, R. (2011). El Período Especial veinte años después. Temas, (65), pp. 59-75.

Espinosa, M. (2009). Arte de conducta. Proyecto pedagógico desde lo artístico. Nueva Ramona. (3), pp. 10-20.

Expósito, M., Vindel, J., \& Vidal, A. (2012). Activismo artístico. En, MNCARS (editores), Perder la forma humana. Una imagen sísmica de los años ochenta en América Latina. pp. 43-50.

Fernández, F. (2003). Complots, del "conflicto" al "sinflicto". Revista Arte Cubano (Dossier), 2, (3), pp. 2-7.

Fornet, A. (2007). El Quinquenio Gris: revisitando el término. Casa de las Américas, (246), p. 3.

Fusco, C. (2017a). Pasos peligrosos: Performance y política en Cuba. Londres: Turner.

Fusco, C. (2017b). En La Habana, una nueva visión para Espacio Aglutinador. The archive. Cuban Art News. Cuban art and culture worldwide. Recuperado de https://cubanartnewsarchive.org/ es/2017/10/10/en-la-habana-una-nueva-vision-para-espacioaglutinador/

González, C. (2017). Cultura autóctona: curaduría como proceso etnográfico en la escena del arte cubano actual. Íconos. Revista de Ciencias Sociales, (59), pp. 55-77.

González, C. (2018). El decreto 349/2018 y la reinvención en la política cultural cubana. Foro Cubano. Vol. 1 (3). Recuperado de https://revistas.usergioarboleda.edu.co/index.php/fc_divul/ issue/view/181

Kocur, Z. (2011). Evolution within the Revolution. The Afro-Cuban Cultural Movement and Cuban Art Collectives, 1975 to 2000. En, J. Harris. (Ed.), Globalization and Contemporary Art (pp. 123136). Reino Unido: Wiley - Blackwell. 
Marrero, R. G. (2021). Mecanismos Represivos del Estado

Cubano. Revista Foro Cubano (RFC), 2(2), pp. 46-58.

Mesa-Lago, C. (1991). El proceso de rectificación en Cuba: causas políticas y efectos económicos. Revista de estudios políticos, (74), pp. 497-532.

Mesa-Lago, C. (2005). Problemas sociales y económicos en Cuba durante la crisis y la recuperación. Revista de la CEPAL. (86), pp. 183-205.

Mosquera, G. (1981). Artes Plásticas: un nuevo salto del venado. Revolución y Cultura, (112), pp. 39-45.

Mosquera, G. (1991). The new art of revolution in Cuba. Art and Text, (39), pp. 22-25.

Mosquera, G. (1998/ 2006). Renovación en los años ochenta. En, M. Espinosa y K. Power. (Eds.), Antología de textos críticos. El Nuevo Arte Cubano. (pp. 17-23). Torrevieja, España: Perceval Press.

Mosquera, G. (1999). Arte y cultura crítica en Cuba. En, I. Anselmi y E. Valdés. (Eds.), La dirección de la mirada: arte cubano contemporáneo. pp. 8-20. Viena: Springer Verlag Wien.

Mora, A. (2017). Arte y educación: identidades en construcción en la Universidad de las Artes, ISA. Revista Clea. (3), pp. 28-44.

Justo, O. (2020). Money Talks: apuntes para un debate sobre el mercado del arte en Cuba. Rialta Magazine. Recuperado de: https://rialta.org/money-talks-mercado-del-arte-en-cuba/

Ojeda, D. (2001/2006). Proyectos-arte en acción de reescritura: La pragmática de René Francisco Rodríguez. En, A. Santana (Ed.). Nosotros, los más infieles: narraciones críticas sobre el arte cubano (1993-2005) (pp. 281-289). Murcia, España: Cendeac.

Parramon, R. (2003). Arte, participación y espacio público. Sublime: arte+cultura contemporánea, (11), 48-51.

Power, K. (1999). Cuba: one history after another. En, P. Pérez y K. Power. (Eds.), While Cuba waits. Art fron the nineties. pp. 23-66. California, EE.UU.: Smart Art Press. 
Power, K. (2006). La claridad en la dicción y el brillo en el naufragio. En, M. Espinosa y K. Power. (Eds.), Antología de textos críticos. El Nuevo Arte Cubano. (pp. 1-4). Torrevieja, España:

Perceval Press.

Ramírez, M. F. (2019). La peña del júcaro martiano: un espacio para el pensamiento libre en el interior de la isla. Foro CubanoDivulgación, 2(15), pp. 1-5.

Saavedra, L. (2014). La pedagogía del arte aplicada a la formación de artistas y la cultura popular. En, W. Canal. (Ed.), Prácticas artísticas e imaginarios sociales. Evento teórico. Oncena Bienal de La Habana. (243-250). La Habana, Cuba: Arte Cubano Ediciones.

Salort, R. C. (2010). La mirada de una isla despierta: dos siglos de arte y enseñanza en Cuba. Bordón. Revista de pedagogía, 62(2), pp. 93-106.

Salort, R.C. (2018). El reto del inédito viable (Desde los réditos contextuales de la relación arte y pedagogía). Artcrónica, (10), pp. 11-17.

Suazo, F. (2018). Arte y política en Cuba en el nuevo milenio. Arte e ideas de América Latina. Recuperado de https://www. coleccioncisneros.org/es/editorial/cite-site-sights/arte-ypol\%C3\%ADtica-en-cuba-en-el-nuevo-milenio

Vázquez Aguiar, D. (2003). Notas para un estudio. El inicio de Enema. Revista Arte Cubano (Dossier), 2, 3, pp. 16-19.

Weiss, R. (2011). To and from Utopia in the New Cuban Art. Minnesota, EE.UU.: University of Minnesota Press.

Wilkinson, S. (2010). La importancia de ser irreverente: ironía, sátira y parodia en las artes visuales cubanas durante el "Período Especial”. En A. Tinajero (Ed.). Cultura y letras cubanas en el siglo XXI. pp. 63-76. Madrid, España: Iberoamericana. 
1. De ahora en adelante también nos referiremos al Instituto Superior de Arte de La Habana con su acrónimo: ISA.

2. El texto completo del manifiesto $27 \mathrm{~N}$ se encuentra disponible en: https://www. cibercuba.com/noticias/2021-04-12-u207888-e207888-s27061-manifiesto-27n-cubarequiere-cambio-ello-necesita

3. Sin pretender hacer una lista exhaustiva, los grupos más representativos que surgen en Cuba de 1980 a 2010 son: Grupo Hexágono, Grupo 4 x 4, Eighteens, Punto, Grupo Provisional, Grupo Independiente La Campana, Art-De, Ponjuán \& Rodríguez, Grupo Pure, Grupo Arte Calle, ABTV, “1, 2, 3, ...12”, Grupo Imán, Galería DUPP (Desde una Pragmática Pedagógica), Los Carpinteros, Gabinete Ordo Amoris, Producciones Doboch, Grupo DIP. (Departamento de Intervenciones Públicas), OMNI Zona Franca y Grupo Enema. 